The second model does not require direct interaction between motors and neurofilaments. Instead, neurofilaments attach directly to microtubules (Fig. 1b). When the microtubule is moved by a dynein- or kinesin-related motor protein, attached neurofilaments are carried along as hitchhikers. This model is consistent with the extensive interactions between microtubules and neurofilaments seen in electron micrographs. Many kinesinrelated proteins and dyneins bind to one microtubule while moving along a second, although no direct evidence exists for concurrent movement of a microtubule and a neurofilament. However, neurofilaments do accumulate in initial segments of axons together with some neurotoxins, and in neuropathologies such as amyotrophic lateral sclerosis, even while microtubule proteins continue into the axon. The reverse situation has not been observed, indicating that neurofilaments may need moving microtubules.

We cannot distinguish between these two models yet. Nonetheless, Wang et al. show clearly that neurofilaments can and do move down axons ${ }^{1}$. The high rates of movement and long pauses between movements of axonal neurofilaments that Wang et al. observed help to reconcile many apparently contradictory results. Experiments such as these provide further insight into how cytoskeletal elements can both move down an axon and provide structural support for that axon at the same time.

Scott T. Brady is at the University of Texas

Southwestern Medical Center

Department of Cell Biology, Room L1.209,

5323 Harry Hines Boulevard, Dallas, Texas

75390-9039, USA.

e-mail:scott.brady@email.swmed.edu

1. Wang, L., Ho, C.-I., Sun, D., Liem, R. K. H. \& Brown, A. Nature
Cell Biol. 2, 137-141 (2000).

2. Baas, P. W. \& Brown, A. Trends Cell Biol. 7, 380-384 (1997).

3. Hirokawa, N., Terada, S., Funakoshi, T. \& Takeda, S. Trends Cell Biol. 7, 384-388 (1997)

4. Brady, S. T., Lasek, R. J. \& Allen, R. D. Cell Motil. 5, 81-101 (1985).

5. Keith, C. H. Science 235, 337-339 (1987).

6. Reinsch, S. S., Mitchison, T. J. \& Kirschner, M. J. Cell Biol. 115, 365-380 (1991)

7. Funakoshi, T., Takeda, S. \& Hirokawa, N. J. Cell Biol. 133, $1347-$ 1353 (1996).

8. Chang, S., Svitkina, T. M., Borisy, G. G. \& Popov, S. V. Nature Cell Biol. 1, 399-403 (1999).

9. Dent, E. W., Callaway, J. L., Szebenyi, G., Baas, P. W. \& Kalil, K. J. Neurosci. 19, 8894-8908 (1999).

10. Yoon, M., Moir, R. D., Prahlad, V. \& Goldman, R. D. J. Cell Biol. 143, 147-157 (1998)

11. Black, M. M., Keyser, P. \& Sobel, E. J. Neurosci. 6, 1004-1012 (1986).

12. Lasek, R. J., Paggi, P. \& Katz, M. J. Brain Res. 616, 58-64 (1993).

13. Prahlad, V., Yoon, M., Moir, R. D., Vale, R. D. \& Goldman, R. D. J. Cell Biol. 143, 159-170 (1998).

14. Elluru, R., Bloom, G. S. \& Brady, S. T. Mol. Biol. Cell 6, 21-40 (1995).

\title{
Zipping up adhesion
}

Cell-cell adhesion is crucial during epithelial development and morphogenesis, with adherens junctions being the main intercellular structures. At each adherens junction, the calcium-dependent celladhesion molecule E-cadherin is directly linked to the actin cytoskeleton through $\alpha$ - and $\beta$-catenin. Other cytoskeletal proteins, including vinculin, $\alpha$-actinin and VASP, have also been implicated, either directly or indirectly, as being part of the adherens-junction structure. During adherens-junction formation, E-cadherin initially localizes to 'punta', formed at the first sites of cell-cell contact. Timelapse imaging and electron-micrographic studies have revealed a subsequent rapid reorganization of the cytoskeletal actin filaments protruding from these puncta, possibly mediated through the GTPase Rho, leading to closure and sealing of the two opposing cell membranes.

Valeri Vasioukhin and colleagues (Cell 100, 209-219; 2000) have now shown that the driving force behind E-cadherin-mediated puncta formation, and rapid actin polymerization, is an increase in calcium levels. Vasioukhin et al. found that, three hours after primary epithelial keratinocytes are moved from low to high levels of extracellular calcium, filopodia - cellular projections - are formed. These filopodia first penetrate, and then embed into, their neighbouring cells. Two opposing rows of E-cadherin (green in the figure opposite) are then seen at these points of contact in punctate pre-adherens junctions. (Arrows in the figure point to so-called adhesion 'zippers'.) Once the puncta are formed, rapid actin polymerization and cytoksleletal reorganization act to pull the opposing membranes together, 'zipping' up the membrane. Desmosomes - structures containing cadherin-family members linked to the intermediatefilament network - then clamp opposing cell membranes at areas unoccupied by adherens junctions. Sealing of the opposing membranes follows.

Vasioukhin et al. go on to show that VASP and MENA, a VASP-family member, are essential for membrane zippering. In cultures of $\alpha$ catenin-deficient keratinocyte cells, there are no E-cadherin zippers, no actin polymerization and, most interestingly, no localization of MENANASP to the punctate adherens-junction structures. The

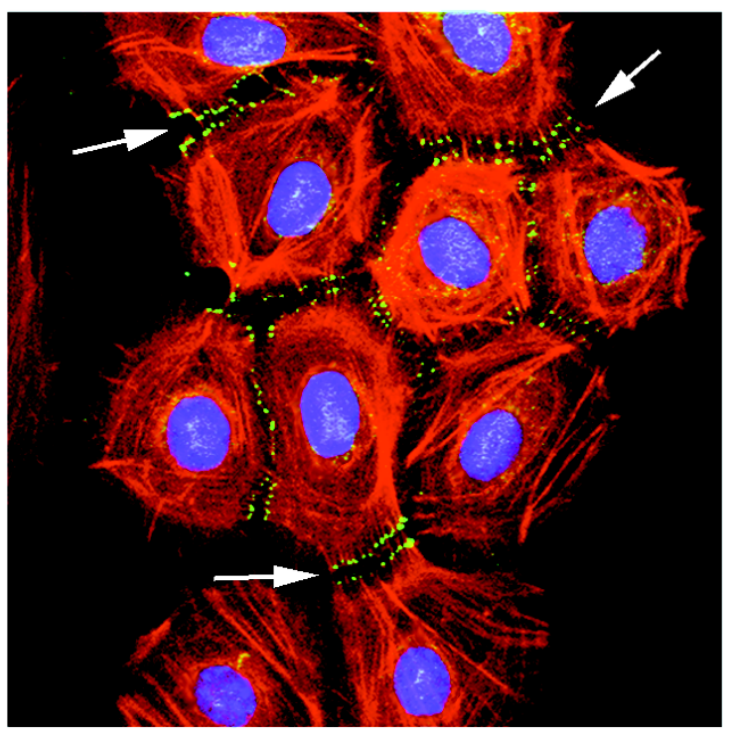

authors therefore propose that $\alpha$-catenin acts to recruit these proteins to the adherens junction, and that it is these proteins, together with vinculin and zyxin, that provide the physical force for rapid actin polymerization and membrane zippering.

It appears that cell-cell adhesion is far from being a 'stick' and 'attach' process: complex interactions between different cytoskeletal proteins are being uncovered. Furthermore, as Ecadherin is a tumour-suppressor protein, loss-of-function mutations in which generate diminished cell-cell adhesion and increased cellular motility, future work should allow us to understand the importance of adhesion regulation and its implications in cellular transformation. 